Musées, Patrimoine et Culture scientifiques et techniques

$168 \mid 2016$

novembre-décembre 2016

\title{
Les risques associés à la conservation des collections militaires et techniques
}

Gilles Aubagnac

\section{OpenEdition \\ Journals}

Édition électronique

URL : http://journals.openedition.org/ocim/1711

DOI : 10.4000/ocim. 1711

ISSN : 2108-646X

Éditeur

OCIM

Édition imprimée

Date de publication : 1 novembre 2016

Pagination : 16-20

ISSN : 0994-1908

Référence électronique

Gilles Aubagnac, "Les risques associés à la conservation des collections militaires et techniques ", La Lettre de l'OCIM [En ligne], 168 | 2016, mis en ligne le 01 novembre 2017, consulté le 19 avril 2019. URL : http://journals.openedition.org/ocim/1711 ; DOI : 10.4000/ocim.1711

Ce document a été généré automatiquement le 19 avril 2019

Tous droits réservés 


\title{
Les risques associés à la conservation des collections militaires et techniques
}

\author{
Gilles Aubagnac
}

Yakovlev 3 (URSS, 1945) exposé dans le hall Normandie Niemen du musée de l'Air et de l'Espace : les risques à connaître et à traiter sont liés aux carburants, lubrifiants, armements.

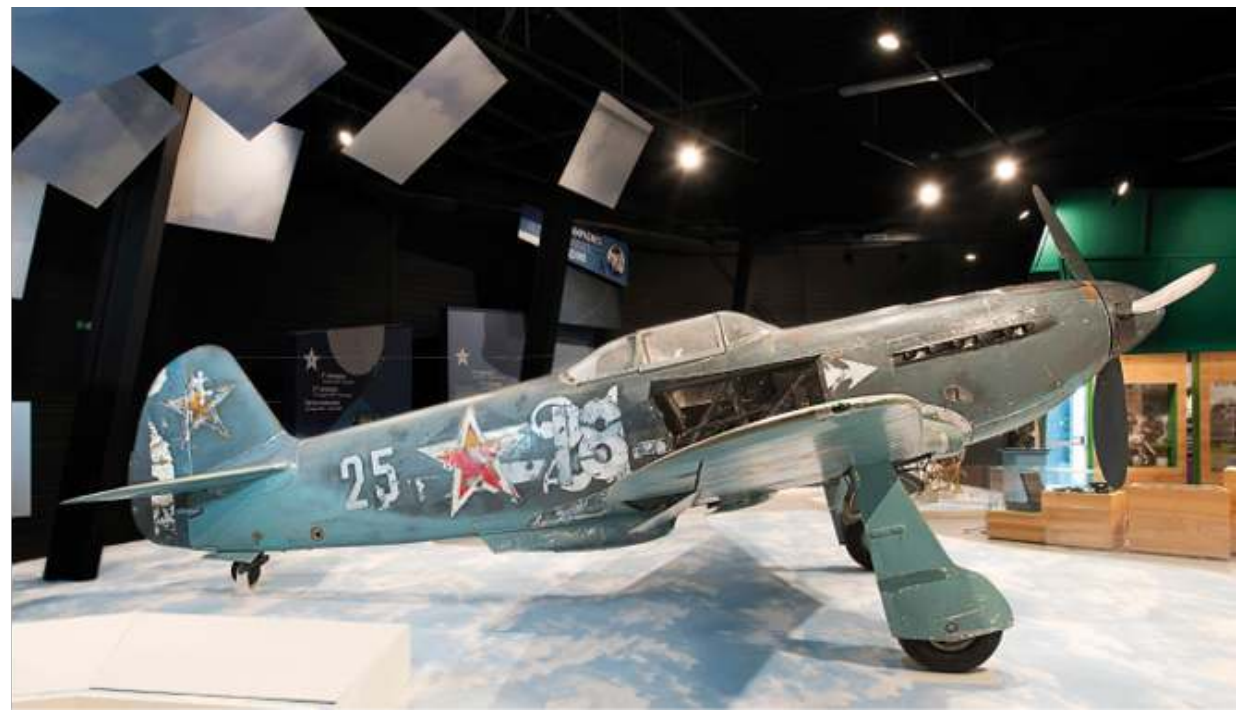

(c) Musée de l'Air et de l'Espace, Le Bourget/F.Cabeza

1 Les matières nucléaires, l'amiante, les carburants et lubrifiants, les munitions et produits explosifs et les armes peuvent être présents dans des collections techniques, industrielles et mécaniques de nombreux musées. Ceci semble assez loin du monde muséal habituel et, parfois, ces questions sont abordées soit avec une certaine indifférence, par méconnaissance du sujet, soit, au contrainte, de manière excessive en raison de peurs, d'émotions ou de réactions instinctives ou subjectives, voire de parti-pris. 


\section{Les matières nucléaires}

2 Le mot fait parfois peur par ce qu'il véhicule donc il est nécessaire d'être précis. Il faut faire la différence entre matière nucléaire et matière radioactive. Ainsi, pour simplifier, il est possible de dire qu'il serait plus dangereux de conserver des échantillons de minéraux radioactifs dans un muséum que les trois matières - tritium, uranium et thorium présentes dans les matériaux des collections techniques et dont il va être question ici ${ }^{1}$.

Il convient néanmoins de prendre des précautions pour la conservation et la manipulation des objets de musée qui contiennent de tels produits. Il est nécessaire aussi de mettre en œuvre, pour certains d'entre-deux, des procédures de contrôle et de sécurité afin d'empêcher les vols car certaines substances pourraient entrer dans la fabrication d'armes "sales" si elles étaient détenues par des groupes terroristes.

\section{Le tritium}

4 Cette matière se trouve principalement dans les objets d'instrumentation comme les cadrans d'horloge, de montre, de boussole, de tableau de bord... Il s'agit de la substance verte ou jaune luminescente qui permet d'utiliser ces équipements ou instruments la nuit ou dans la pénombre. Le tritium a été largement utilisé jusqu'à la fin du $\mathrm{XX}^{\mathrm{e}}$ siècle et se retrouve aujourd'hui dans de nombreuses collections de musée. Il ne présente pas de danger particulier lors d'exposition et de manipulation sauf si le boîtier et/ou le verre de protection est absent ou cassé (risque de poussière) ou s'il est stocké en très grande quantité dans un lieu unique et clos. Il n'existe pas de contrôle réglementaire à mettre en œuvre pour ce produit et les objets qui en contiennent.

\section{L'uranium}

5 Cette matière, citée ici de manière générique, est présente dans de nombreux matériels médicaux, de laboratoire, mais aussi dans des moteurs, des engins de transport ferroviaire, des avions... En effet, l'uranium est très dense : 1 mètre cube d'uranium pèserait de l'ordre de 19 tonnes. Ceci permet l'alourdir un objet de manière significative tout en lui gardant de petites dimensions ; ainsi les gouvernes de profondeur du Boeing 747 contiennent de l'uranium. Cette matière se trouve surtout dans les musées aéronautiques conservant des aéronefs de la deuxième moitié du $\mathrm{XX}^{\mathrm{e}}$ siècle. Généralement, la présence d'uranium est mentionnée dans la documentation technique.

6 Ces matières nucléaires sont suivies par l'Institut de radioprotection de sureté nucléaire $(\text { IRSN })^{2}$. Elles doivent faire l'objet, par le musée détenteur, d'une inscription dans un registre journal dans lequel sont indiqués les mouvements des objets : entrée dans les collections, prêts, restauration... Un compte rendu annuel doit être fait à l'IRSN.

\section{Le thorium}

7 Il est présent dans de nombreuses collections techniques et modernes de musées sous forme d'alliage mais en dose de peu d'importance. La soudure à l'argon ou l'électricité se fait avec des baguettes de tungstène associé à du thorium ce qui explique la présence de ce dernier dans les moteurs, les carrosseries, les équipements mécaniques et techniques. 
À partir des années 1970, la présence de thorium est généralement signalée dans les documents techniques.

8 Le thorium ne présente pas un danger particulier pour le personnel ou le visiteur. Il y a néanmoins une obligation de tenir un registre-journal et, au-delà d'un certain seuil, de transmettre un compte-rendu annuel à l'IRSN. Le musée de l'Air et de l'Espace, qui possède une collection de quatre cents aéronefs et de centaines de moteurs, n'a pas atteint ce seuil.

Caméra américaine K37 avec objectif Aero-Ekta pour avion : contient de l'oxyde de thorium.

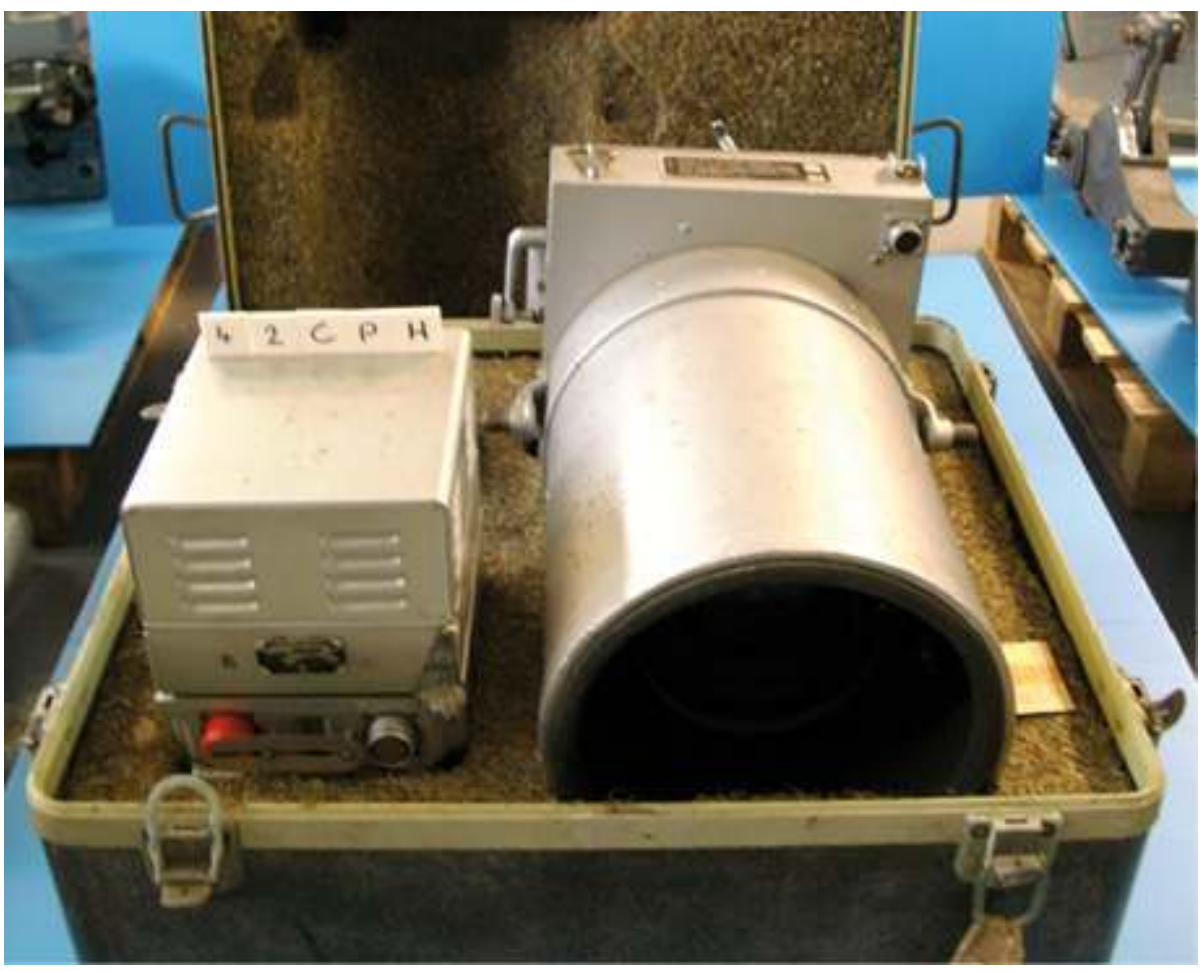

(c) Musée de l'Air et de l'Espace, Le Bourget

9 Les trois matières nucléaires font l'objet de textes réglementaires qu'un musée possédant des collections de ce type se doit de connaitre ${ }^{3}$ :

10 - le décret 81-512 du 22 mai 1981 qui donne les définitions nécessaires;

11 - les articles L 1333-1 à 14 du Code de la Défense qui traitent des autorisations, des contrôles et des sanctions ;

12 - l'arrêté du 14 mars 1984 (modifié le 21 mai 2003) qui traite des déclarations, du livrejournal et de l'inventaire annuel. 


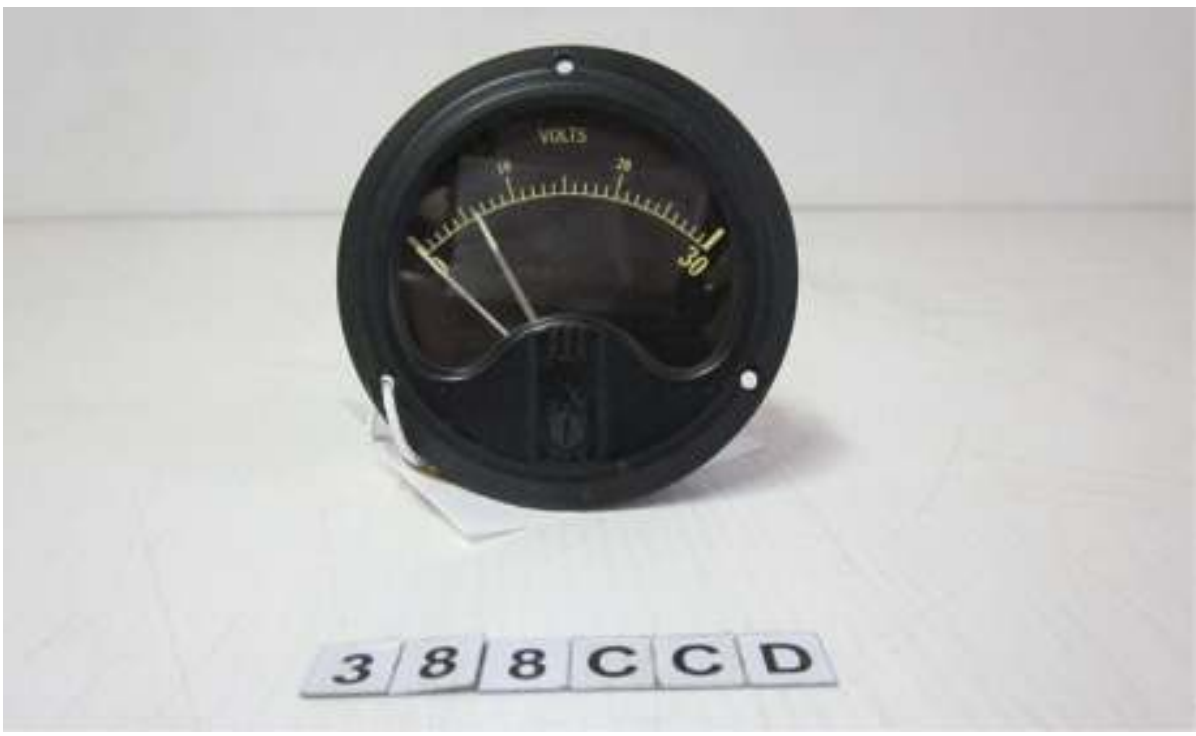

(C) Musée de l'Air et de l'Espace, Le Bourget

\section{L'amiante}

La question de la gestion des collections contenant de l'amiante est aujourd'hui l'une des plus sensibles qui se pose aux responsables de musée. Au-delà des aspects réglementaires, le mot lui-même, subjectivement, entraîne ou entretient une certaine peur ; il faut donc aller au-delà.

Le texte-clé en matière d'amiante est le décret n96-1133 du 24 décembre 1996. L'article 1 er est très clair et interdit "[...] la détention en vue de la vente et la cession à quelque titre que ce soit de toutes variétés de fibres d'amiante et de tout produit en contenant".

La conservation et la gestion de ces collections relèvent des préconisations et des obligations définies par l'Institut national de recherche et de sécurité (INRS) et le code du Travail y fait référence.

16 Un mémorandum sur l'amiante et la conservation du patrimoine culturel a été établi en 2014 par la Direction générale des patrimoines du ministère de la Culture (Service du Patrimoine/SDMHEP Service des Musées de France/C2RMF) ainsi que des propositions d'évolution de la réglementation dans le respect de la sécurité des personnes.

17 L'amiante est présent dans les collections liées aux moyens de transports (voitures, camions, tracteurs, locomotives et wagons, aéronefs...) dans les isolants des contacteurs, les joints de culasse, les garnitures de freins, les protections contre la chaleur et l'incendie (équipements de pompiers) mais aussi parfois dans des accessoires de théâtre, des objets liés à la verrerie, la fonderie, la métallurgie, tout comme dans des sèchecheveux ou des vêtements funéraires et même dans certaines céramiques corses du XIX siècle.

En règle générale, le public est peu concerné par la présence de l'amiante. En revanche, le personnel d'entretien des collections et les restaurateurs sont particulièrement 
concernés. L'IRSN a diffusé une information et un protocole pour les situations de travail exposant à l'amiante ${ }^{4}$.

19 En cas de doute sur la présence d'amiante dans des collections, il convient de faire réaliser une expertise. Dans certains cas, le Laboratoire de recherche des monuments historiques (LRMH) est en mesure de la réaliser ${ }^{5}$.

20 Les protocoles de travail relatif au conditionnement, à la présentation au public, aux qualifications et à la formation des intervenants pour les collections susceptibles de contenir de l'amiante sont encore embryonnaires mais des textes réglementaires devraient voir le jour dans un avenir plus ou moins proche.

\section{Les carburants et lubrifiants}

21 Il est évident qu'il ne faut jamais laisser de carburant (essence, gasoil, kérosène) dans les moteurs aussi bien pour les collections exposées qu'en réserve. Les réservoirs doivent donc être vidangés mais il faut aussi réaliser un dégazage de ceux-ci car les vapeurs d'essence sont très dangereuses.

La question des lubrifiants est plus complexe. Une couche de lubrifiant dans les mécanismes d'un moteur constitue une excellente protection pour la corrosion. Mais ceci n'est vrai que pour du court terme car à la longue les huiles et graisses deviennent pâteuses puis durcissent. S'il n'y a pas de protection et si les conditions de conservation ne sont pas draconiennes (en particulier température et humidité relative) la corrosion s'installe et pour la traiter il faut démonter les mécanismes. Les enjeux et les solutions deviennent alors complexes.

Un des bâtiments abritant les réserves du musée de l'Air et de l'Espace : la vidange et le dégazage sont des opérations primordiales dans des réserves de collections de ce type.

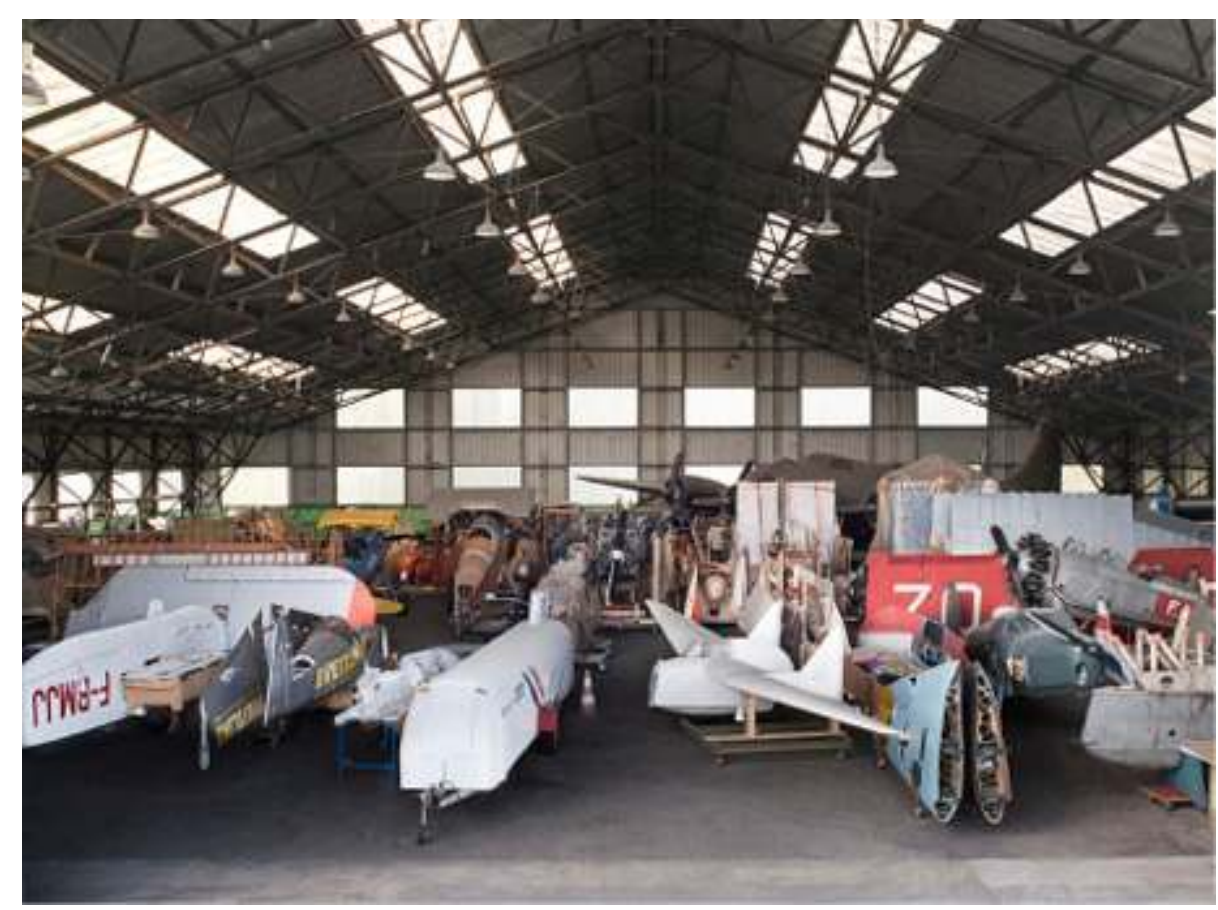

(C) Musée de l'Air et de l'Espace, Le Bourget/F. Cabeza 
4 août 1789, en détenir une devient le symbole de la Liberté et de l'Égalité. Chaque citoyen pouvait être propriétaire d'armes à feu puisque la défense de la Nation reposait sur la volonté des citoyens et la chasse n'était plus le privilège de la noblesse. Il en est ainsi durant tout le XIX siècle et le premier tiers du XXe siècle. Dans les années 1930, la peur d'une insurrection armée, avec les ligues à droite et le Parti Communiste à gauche amène le gouvernement à promulguer le décret-loi du 18 avril 1939 qui réglemente, pour la première fois, le droit de posséder une arme de guerre et définit des types d'armes différents : de guerre, de chasse, de tir, d'épaule, de poing... Depuis, ces textes ont été renforcés à maintes reprises $(1973,1976,1983,1987,1993,1995,1998 . .$.$) ; la dernière loi$ date de 2013.

Aujourd'hui, la détention d'armes est codifiée par le décret n²013-700 du 30 juillet 2013 portant application de la loi n²012-304 du 6 mars 2012 relative à l'établissement d'un contrôle des armes "moderne, simplifié et préventif"7.

Selon l'article L2331-1 du Code de la Défense les armes sont classées en diverses catégories qui définissent les conditions de détention. Catégorie $\mathrm{A}$ : matériels de guerre et armes interdits à l'acquisition et à la détention, sou réserve des dispositions des articles L. 312-1 à L. 312-4-3 du Code de la sécurité intérieure. Cette catégorie comprend : les armes et éléments d'armes interdits à l'acquisition et à la détention ; les armes relevant des matériels de guerre, les matériels destinés à porter ou à utiliser au combat les armes à feu, les matériels de protection contre les gaz de combat ;

Catégorie $\mathrm{C}$ : armes soumises à déclaration pour l'acquisition et la détention ;

Catégorie D : armes soumises à enregistrement et armes et matériels dont l'acquisition et la détention sont libres :

- les armes historiques et de collection dont le modèle est antérieur au $1^{\mathrm{er}}$ janvier 1900 (à l'exception de celles classées dans une autre catégorie en raison de leur dangerosité), les 
munitions et éléments de munitions à poudre noire utilisables dans les armes historiques et de collection;

\section{neutralisés.}

Nieuport XI équipé d'une mitrailleuse Lewis à chargeur circulaire (1916).

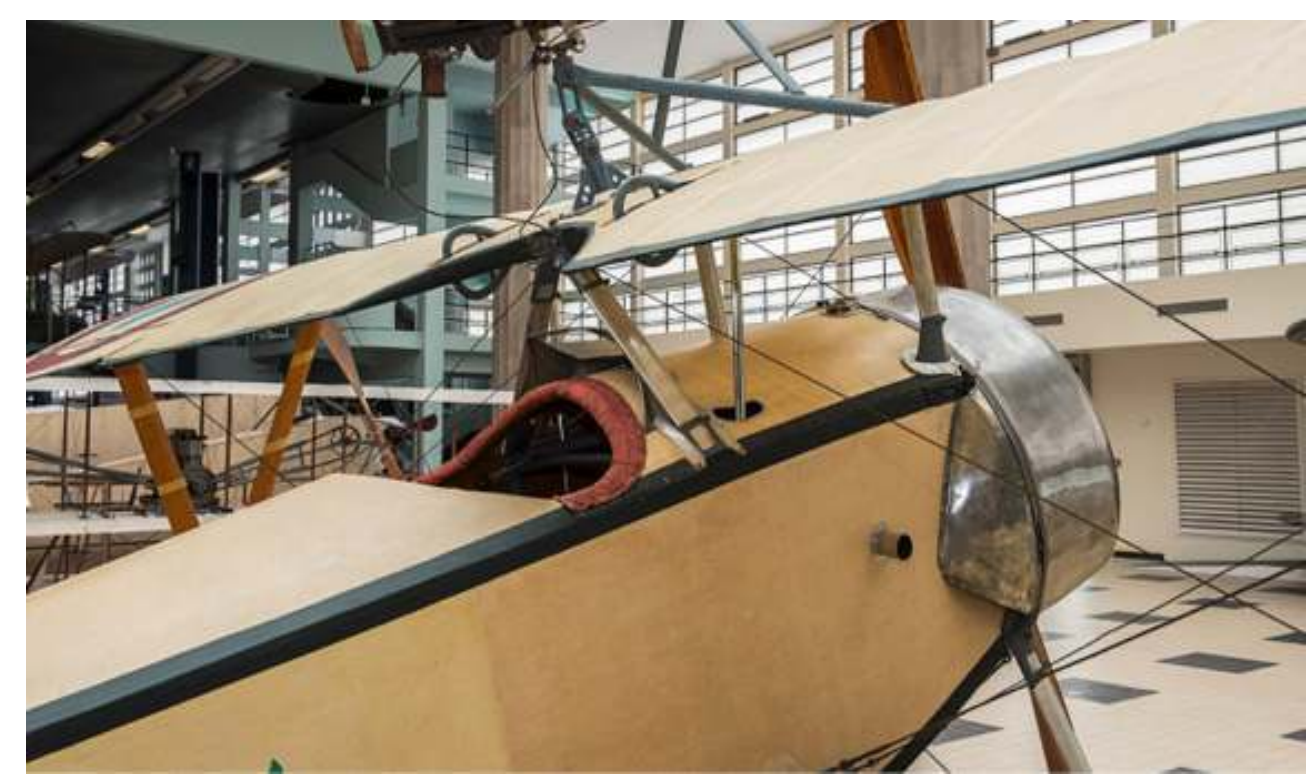

(C) Musée de l'Air et de l'Espace,Le Bourget/F. Cabeza

La catégorie D concerne donc les musées qui conservent des armes dont le modèle est antérieur à 1900. Il ne faut pas confondre modèle et année de fabrication. Ainsi, un fusil Lebel de la Première Guerre mondiale qui porte, par exemple, l'année 1915 marquée à froid est bien du modèle antérieur à 1900 puisque ce type de Lebel est du modèle 1886, modifié 1893. Il n'est donc pas nécessaire de neutraliser une telle arme. Pour les armes plus récentes, cet article prévoit une neutralisation. Cette opération rend l'arme irrévocablement impropre au tir mais elle est surtout une atteinte à l'intégrité physique de l'objet. Ceci est contraire au Code du Patrimoine et à la déontologie. La législation des armes évolue. Il y a quelques années le révolver d'ordonnance modèle 1874 devait être neutralisé. Ce n'est plus le cas aujourd'hui. Il convient donc de ne pas injurier l'avenir et le législateur a rendu possible, dans certaines conditions, la possession d'armes non neutralisées dans les collections de musée. L'article 27 de la loi est à connaître par les responsables des collections d'armes dans les musées classés ou non Musée de France :

I/ Peuvent être autorisés, par le préfet sur avis du ministre de la défense 8 , sous réserve, pour les personnes physiques, des dispositions de l'article 13, à acquérir et à détenir des matériels de guerre, armes, munitions et leurs éléments :

$1^{\circ}$ Les personnes qui les exposent dans des musées, ouverts au public, pour les matériels de guerre, armes et leurs éléments ainsi que les munitions de toutes catégories;

$412^{\circ}$ Les services de l'État, pour les besoins autres que ceux de la défense nationale, les collectivités territoriales et leurs établissements publics, pour les matériels de guerre de la catégorie A et les armes des catégories $A$ et $B$; 
$3^{\circ}$ Les organismes d'intérêt général ou à vocation culturelle, historique ou scientifique, qui contribuent à la conservation, à la connaissance ou à l'étude des matériels de guerre, pour les matériels de la catégorie $A$ et les armes des catégories $A$ et $B$;

$4^{\circ}$ Les personnes physiques qui contribuent, par la réalisation de collections, à la conservation, à la connaissance ou l'étude des matériels de guerre, pour les matériels de la catégorie A2;

$5^{\circ}$ Les établissements d'enseignement et de formation, en vue de l'accomplissement de leur mission, pour les matériels de guerre relevant des $8^{\circ}, 9^{\circ}$ et $10^{\circ}$ de la catégorie $\mathrm{A} 2$.

II/ Sauf pour les prototypes, les autorisations d'acquisition et de détention des matériels de guerre de la catégorie A2 mentionnés au I ne peuvent être accordées aux demandeurs mentionnés aux $2^{\circ}$, $3^{\circ}$ et $4^{\circ}$, pour un matériel donné, que si le premier exemplaire du même type a été mis en service trente ans au moins avant la date de dépôt de la demande d'autorisation et si la fabrication du dernier exemplaire du même type a été arrêtée vingt ans au moins avant cette même date. l'article 22, l'autorisation d'acquisition et de détention des matériels de guerre mentionnés au I est accordée sans limitation de durée. Lorsque l'autorisation porte sur un matériel de guerre des $8^{\circ}, 9^{\circ}$ et $10^{\circ}$ de la catégorie $\mathrm{A} 2$, son titulaire est tenu de signaler tout changement du lieu de détention de ce matériel au préfet du département de l'ancien et du nouveau lieu de détention.

47 Dans un musée, le risque majeur serait certes le vol. Néanmoins, depuis la fin du XXe siècle avec les guerres dans les Balkans et l'effondrement du Bloc de l'Est, il est plus facile de se procurer une Kalachnikov en état de tir avec des munitions qu'un FSA MAS 49-56 conservé dans un musée?.

Moteur Rolls Royce Trend 900 pour Airbus A382, exposé dans le hall Concorde du musée de l'Air et de l'Espace.

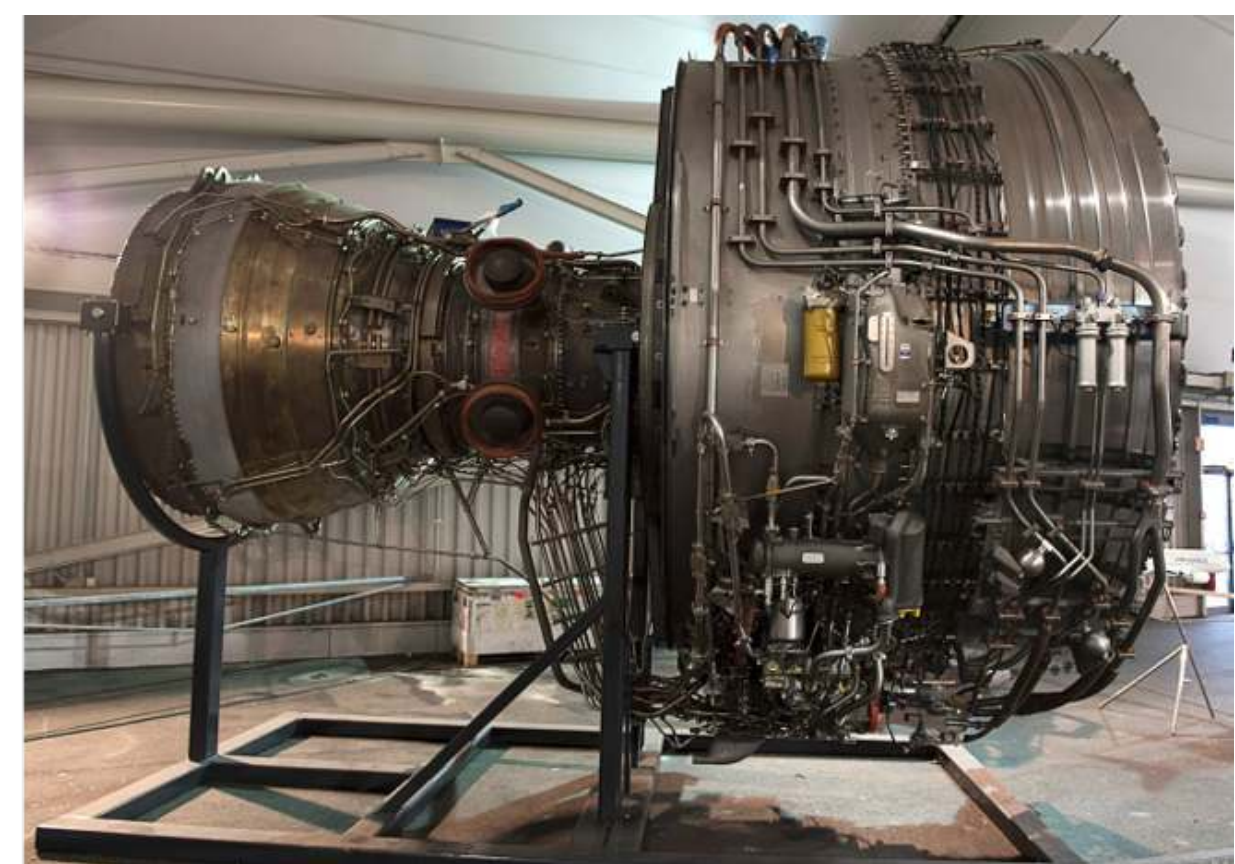

(C) Musée de l'Air et de l'Espace, Le Bourget/F. Cabeza.

Les risques associés à la conservation des collections techniques comme les armes, les munitions et matériels explosifs et tous les objets contenant des matériaux toxiques (matières nucléaires, amiante, carburants et lubrifiants) sont réels. Toutefois, il convient 
d'aborder ces questions en évitant l'émotion et la polémique. Des textes réglementaires existent, des protocoles d'action sont définis ou en voie de l'être. Il revient donc au responsable de collections de ce type de les connaître et de toujours déployer une analyse à la fois à la lettre et dans l'esprit car chaque objet de musée est un cas unique même pour des collections de type industriel.

\section{NOTES}

1. Minéraux radioactifs, des collections à sécuriser, Repères (magazine d'information de l'IRSN), $\mathrm{n}$ ${ }^{\circ} 27$, décembre 2015, pp. 6-8.

2. www.irsn.fr/FR/Pages/Home.aspx Minéraux radioactifs, des collections à sécuriser

3. Ces textes peuvent facilement être trouvés sur Legifrance : www.legifrance.gouv.fr/

4. Situations de travail exposant à l'amiante; www.inrs.fr/media.html?refINRS=ED\%206005

5. Laboratoire de recherche des monuments historiques, 29 rue de Paris, 77420 Champs-surMarne.

6. Voir par exemple : www.orne.gouv.fr/IMG/pdf/Deminage Que faire 61 cle6666f7.pdf ou www.deminex.fr/

7. Décret $n^{\circ} 2013-700$ du 30 juillet 2013 portant application de la loi n²012-304 du 6 mars 2012 relative à l'établissement d'un contrôle des armes moderne, simplifié et préventif (JORF n0178 du 2 août 2013) www.legifrance.gouv.fr/affichTexte.do? cidTexte=JORFTEXT000027792819\&categorieLien=id

8. Les expressions et phrases soulignées le sont par l'auteur de cet article.

9. Fusil semi-automatique de la manufacture d'armes de Saint-Étienne modèle 1949 modifié en 1956, en dotation dans l'armée française durant quasiment toute la deuxième moitié du XX siècle

\section{RÉSUMÉS}

Les risques associés à la conservation des collections militaires et techniques (munitions et matériels explosifs, objets contenant des matériaux toxiques) peuvent paraître nombreux et parfois complexes : à partir de cinq cas principaux (matières nucléaires, amiante, carburants et lubrifiants, munitions et produits explosifs et armes), l'auteur précise les différents textes réglementaires en vigueur et livre plusieurs préconisations et protocoles d'action.

\section{INDEX}

Mots-clés : collection militaire, collection technique, risque 


\section{AUTEUR}

\section{GILLES AUBAGNAC}

conservateur, chef du service des collections au musée de l'Air et de l'Espace, Le Bourget gilles.aubagnac@museeairespace.fr 